\title{
Maternal periodontitis decreases plasma membrane GLUT4 content in skeletal muscle of adult offspring
}

\author{
Maria Sara de Lima Coutinho Mattera ${ }^{a}$, Fernando Yamamoto Chiba ${ }^{b}$, Max Sander de Oliveira da Mota ${ }^{a}$, \\ Renato Felipe Pereira ${ }^{a}$, Edilson Ervolino ${ }^{a}$, Antonio Hernandes Chaves Neto ${ }^{a}$, Leda Maria Pescinini Salzedas ${ }^{\text {, }}$ \\ Natália Francisco Scaramele ${ }^{\mathrm{a}}$, Cristina Antoniali Silva ${ }^{\mathrm{a}}$, Maristela Mitiko Okamoto ${ }^{\mathrm{d}}$, \\ Ubiratan Fabres Machado ${ }^{\mathrm{d}}$, Doris Hissako Sumida ${ }^{\mathrm{a}, *}$ \\ a Programa de Pós-graduação Multicêntrico em Ciências Fisiológicas-SBFis - Department of Basic Sciences, Dental School of Araçatuba, Univ Estadual Paulista - UNESP, Araçatuba, SP, Brazil \\ ${ }^{\mathrm{b}}$ Preventive and Social Dentistry Post-Graduation Program, Dental School of Araçatuba, Univ Estadual Paulista - UNESP, Brazil \\ c Department of Pathology and Clinical Propedeutic, Dental School of Araçatuba, Univ Estadual Paulista - UNESP, Brazil \\ d Department of Physiology and Biophysics, São Paulo Institute of Biomedical Sciences, USP, Brazil
}

\section{A R T I C L E I N F O}

\section{Article history:}

Received 9 October 2015

Received in revised form 4 February 2016

Accepted 4 February 2016

Available online 23 February 2016

\section{Keywords:}

Periodontal disease

Diabetes mellitus

Glucose transporter type 4

Low birth weight

Protein kinase B

\begin{abstract}
A B S T R A C T
Aims: The fetal programming hypothesis suggests that intrauterine stimuli can induce metabolic changes in offspring, increasing the disease risk in adulthood. Periodontal disease may enhance serum cytokine levels. Cytokines such as tumor necrosis factor-alpha (TNF- $\alpha$ ) have been associated with reduced glucose transporter type 4 (GLUT4) expression, decreased protein kinase B (Akt) phosphorylation, and insulin resistance. This study aimed to evaluate GLUT4 content, and Akt serine phosphorylation status in the gastrocnemius skeletal muscle (GSM), glycemia, insulinemia and change in body weight in offspring of rats with periodontal disease.

Main methods: Female Wistar rats were distributed into a control group $(\mathrm{CN})$ and an experimental periodontal disease group (PD), in which a ligature was placed around the mandibular first molars. Seven days after ligature placement, both groups were mated with normal male rats. The ligatures remained throughout pregnancy until weaning, after which the male offspring were distributed into groups: $\mathrm{CN}-\mathrm{O}$, control rat offspring; and PD-o, periodontal disease rat offspring. The body weight from 0 to 75 days of age was measured. At 75 days, the glycemia, insulinemia, TNF- $\alpha$ levels, Akt serine phosphorylation, and GLUT4 content in the GSM were measured in the offspring.

Key findings: The PD-o group showed a low birth weight (LBW), unchanged glycemia, increased insulinemia, insulin resistance, increased TNF- $\alpha$ levels, decreased Akt serine phosphorylation status, and reduced GLUT4 content in the plasma membrane and translocation index after insulin stimulation.

Significance: Maternal periodontal disease causes LBW, insulin resistance, and alterations in the final stage of insulin signaling in the GSM of adult offspring.
\end{abstract}

C 2016 Elsevier Inc. All rights reserved.

\section{Introduction}

Periodontal disease is a local inflammatory process characterized by the release of toxic products, such as lipopolysaccharides, by pathogens and the host immune response comprising neutrophil, macrophage, and lymphocyte infiltration, and the release of cytokines such as tumor necrosis factor-alpha (TNF- $\alpha)[1]$.

During pregnancy, periodontal pathogens in the oral cavity can reach the amniotic cavity and cause adverse outcomes such as low birth weight (LBW) [2]. There is a correlation between LBW and an

\footnotetext{
* Corresponding author at: Dental School of Araçatuba, UNESP - Univ Estadual Paulista, R. José Bonifácio, 1193, CEP: 16015-050 Araçatuba, SP, Brazil.

E-mail address: dorishs@foa.unesp.br (D.H. Sumida).
}

increased predisposition to hypertension, coronary heart disease, and diabetes in adulthood [3-5]. This is explained by the fetal programming mechanism, in which environmental stimuli within the uterus affect fetal growth and health, not only during gestation, but also during adult life $[4,6,7]$.

Pregnant women with periodontitis showed increased serum TNF- $\alpha$ and interleukin (IL)-6 concentrations [8]. Excessive TNF- $\alpha$ concentration causes insulin resistance by modifying the insulin signal transduction [9]. Insulin binds the $\alpha$-subunit of its receptor, which triggers autophosphorylation of the $\beta$-subunit followed by stimulation of tyrosine kinase activity, tyrosine phosphorylation of the insulin receptor substrate (IRS), and recruitment of phosphatidylinositol 3-kinase (PI3K). Ultimately, this pathway triggers activation of several serine/ threonine kinases, most notably protein kinase B (PKB/Akt), which 
enables glucose uptake into the cells by translocating the glucose transporter type 4 (GLUT4) from the intracellular compartment to the plasma membrane $[10,11]$.

Shirakashi et al. [12] showed that maternal periodontal disease altered the initial steps of the insulin-signaling pathway, as indicated by a reduction in pp185 tyrosine phosphorylation in the gastrocnemius skeletal muscle (GSM) and white adipose tissue.

Therefore, this study evaluated the relationship between maternal periodontal disease, LBW, and alterations in the final stage of insulin signaling in the GSM of their offspring, as abnormal insulin activity in muscle represents an early marker for an increased risk of diabetes.

\section{Materials and methods}

\subsection{Animals}

This study followed the Ethical Principles and Guidelines for Animal Experimentation, and was approved by the local ethics committee (protocol number 00863-2013). The Wistar rats were housed with a 12-h light/dark cycle (lights on at 07:00), ambient temperature of $23 \pm$ $2{ }^{\circ} \mathrm{C}$, and food (Presence ${ }^{\circledR}$, Paulínia, São Paulo, Brazil) and water available ad libitum. The diet (3.6 kcal/g-digestible energy) contained an average macronutrient composition as follows: $23 \%$ from protein, $4 \%$ fat and 58\% from carbohydrate (Presence®, Paulínia, São Paulo, Brazil).

\subsection{Experimental design}

Sixteen Wistar female rats (approximately $200 \mathrm{~g}$ each) were randomly divided into two groups: $\mathrm{CN}$, control group $(n=8)$; and PD, experimental periodontal disease group $(n=8)$. In the PD group, periodontal disease was induced by installing a ligature with 4-0 silk (Seda-Silk, Johnson \& Johnson, São José dos Campos, Brazil) thread onto the cervical region of the mandibular first molars bilaterally, as described by [13]. Seven days later, the rats in both groups were mated with normal male rats. Vaginal smears were taken daily to verify the presence of sperm. After confirming pregnancy, the female rats were housed individually. The food intake and body weight of the mother rats were evaluated weekly during the periods of pregnancy and lactation. The ligature was maintained throughout pregnancy until weaning the offspring.

After birth, the numbers of offspring in the $\mathrm{CN}$ and PD groups were matched so that each mother had the same number of offspring. After weaning, the adult female rats were euthanized (thiopental overdose, $150 \mathrm{mg} / \mathrm{kg}$ ), and the male offspring were studied to avoid any effect from hormonal oscillation during the estrous cycle in the juvenile female rats. The male offspring were distributed into two groups: $\mathrm{CN}-$ 0 , which were the offspring of the control rats $(n=16)$; and PD-o, which were the offspring of the rats with experimental periodontal disease $(n=16)$. The body weights of the offspring were measured once weekly from birth until 75 days of age, and experiments were performed once the offspring were 75 days old. Food intake of the $\mathrm{CN}-\mathrm{O}$ and PD-o groups was evaluated weekly from weaning until the end of the experiment.

Animals were fasted for $14 \mathrm{~h}$ prior to experiments and anesthetized with sodium thiopental (Thiopentax ${ }^{\circledR}$, Cristália, Itapira, Brazil) (3\%, $50 \mathrm{mg} / \mathrm{kg}$, intraperitoneal). Experiments were performed 10-15 min later.

A median laparotomy was performed in rats from the $\mathrm{CN}-\mathrm{o}(n=8)$ and PD-o $(n=8)$ groups, and blood samples $(4 \mathrm{~mL})$ were taken from the inferior vena cava. The blood samples were then transferred into tubes containing heparin (Liquemine, Hoffmann-La Roche, Basle, Switzerland). Plasma was prepared by centrifuging the blood at $3000 \times \mathrm{g}$ for $15 \mathrm{~min}$ at $4{ }^{\circ} \mathrm{C}$, and stored in aliquots at $-70{ }^{\circ} \mathrm{C}$ until glucose, insulin and TNF- $\alpha$ quantification.

The periepididymal white adipose tissue and left GSM were collected, and the absolute weight $(\mathrm{g}$ ) and relative weight (per $100 \mathrm{~g}$ of body weight) of these tissues $(n=8)$ were measured. The left GSM ( $n=6)$ was used to measure the GLUT4 protein content. After this procedure, the animals were euthanized. Finally, the remaining animals from the $\mathrm{CN}-\mathrm{O}(n=8)$ and PD-o $(n=8)$ groups were used to evaluate the Akt serine phosphorylation status in the GSM following insulin (Novolin ${ }^{\circledR}$, Novo Nordisk, Bagsvaerd, Dinamarca) administration (1.5 $\mathrm{U}$, intravenously into the portal vein).

\subsection{Radiographic and histological analysis of maternal periodontal tissues}

The periodontal health (CN group) or effectiveness of periodontal disease induction (PD group) was confirmed using radiographic and histological analysis of the maternal jaws.

The adult female rats were euthanized after weaning the offspring, and the right and left hemi-mandibles were dissected and fixed for $24 \mathrm{~h}$ in $4 \%$ formaldehyde. The mandibles were radiographed at a $70 \mathrm{kvp}, 10 \mathrm{~mA}$, and $0.10 \mathrm{~s}$ exposure. The source-to-film distance was always set at $40 \mathrm{~cm}$. The digital image was obtained directly with an optical digital plate (Digora, Soredex, Orion Corporation, Helsinki, Finland), and the optical plates were read in sensitized laser scanner equipment (Windows Digora 1.51, Soredex, Orion Corporation, Helsinki, Finland). The images were analyzed to verify the presence of alveolar bone loss in the first molars.

After obtaining the radiographic images, the hemi-mandibles were demineralized in 10\% EDTA (UltraPureTM EDTA, Invitrogen, Scotland, UK) ( $\mathrm{pH}$ 7.4) for 3 months. The specimens were processed conventionally and embedded in paraffin. Semi-serial sections ( $5 \mu \mathrm{m})$ were obtained in the distal-mesial direction and stained with hematoxylin and eosin (H\&E). Histological analysis was performed as described by Garcia et al. [14]. A certified histologist (EE) evaluated to the following parameters: nature and degree of inflammation; extent of the inflammatory process; presence and extent of tissue necrosis; presence, extent, and nature of bone, cementum, and dentin resorption; state of the vasculature; extracellular matrix structure of the periodontal tissues; and cellularity pattern of the periodontal tissues.

\subsection{Glycemia, insulinemia, HOMA-IR index and plasma concentration of TNF- $\alpha$ in offspring}

Using the 8 plasma samples in each group ( $\mathrm{CN}-\mathrm{o}$ and $\mathrm{PD}-\mathrm{o})$, the glucose concentrations were measured by the glucose oxidase method (Enzymatic glucose, ANALISA Diagnóstica, Belo Horizonte, MG, Brazil), and insulinemia was measured by radioimmunoassay (Sensitive Rat Insulin, SRI-13K, Millipore, St Charles, MO, EUA). Insulin resistance was evaluated by the HOMA-IR index (homeostasis model assessment of insulin resistance), which was calculated according to the following formula: HOMA-IR = fasting glycemia $(\mathrm{mmol} / \mathrm{L}) \times$ fasting insulinemia $(\mu \mathrm{IU} / \mathrm{mL}) / 22.5[15,16]$. Plasma concentrations of TNF- $\alpha(n=8)$ were quantified using an enzyme-linked immune sorbent assay (ELISA) kit (Invitrogen Corporation, Camarilio, CA, USA).

\subsection{Evaluation of Akt serine phosphorylation status in skeletal muscle of offspring}

The GSM was collected from eight animals in each group ( $\mathrm{CN}-\mathrm{o}$ and $\mathrm{PD}-\mathrm{o}$ ) before and $90 \mathrm{~s}$ after administering $1.5 \mathrm{U}$ of regular insulin (intravenously through the portal vein). The tissue samples were prepared as described by Carvalho et al. [17] and the Akt serine phosphorylation status following insulin stimulus was quantified using Western blotting and antiphosphoserine AKTSer473 antibody (Santa Cruz Biotechnology Inc., Santa Cruz, CA, USA).

The total Akt was quantified using Western blotting and antitotal Akt antibody (Santa Cruz Biotechnology, Inc, Santa Cruz, CA, USA) and $\beta$-actin (Santa Cruz Biotechnology Inc., Santa Cruz, CA, USA) served as the loading control. Immunoreactive bands were detected by autoradiography using a chemiluminescent substrate system (GE Healthcare, 
Buckinghamshire, UK), according to the manufacturer's instructions. The blots were quantitatively analyzed using software (Scion ImageRelease Beta 3b, NIH, Frederick, MD, USA).

\subsection{GLUT4 protein analysis in offspring}

Tissue samples were homogenized in sucrose buffer $\mathrm{pH} 7.4$ ( $10 \mathrm{mmol} / \mathrm{L}$ Tris- $\mathrm{HCl}, 1 \mathrm{mmol} / \mathrm{L}$ EDTA, and $250 \mathrm{mmol} / \mathrm{L}$ sucrose) and subjected to differential centrifugations to obtain the plasma membrane (PM) and microsomal-enriched (M) fractions of the GSM [18]. Equal amounts of the membrane and microsomal-enriched protein fractions were resolved on a $10 \%$ SDS gel. The blots were quantified by densitometry (ImageQuant TL, GE Healthcare UK Limited, Buckinghamshire, UK). In the GLUT4 protein analysis, the Ponceau stained membrane was used as a loading control. The translocation index was calculated as follows: Translocation index of GLUT4 $=\mathrm{PM} \times 100 /(\mathrm{PM}+\mathrm{M})$ [19].

\subsection{Statistical analysis}

The body weight and food intake of the mother rats; body weight at 75 days, food intake and the Akt serine phosphorylation status in offspring were assessed by analysis of variance, followed by the Holm Sidak post-hoc test when the analysis of variance suggested a significant difference between the groups $(p<0.05)$.

The birth weight, periepididymal white adipose tissue and GSM weights, glycemia, insulinemia, HOMA-IR, and the GLUT4 content in the $\mathrm{M}$ and PM fractions and its translocation index were analyzed using the Student $t$-test. A $p$-value of $<0.05$ was considered statistically significant.

The normality of the dataset was verified for all analyses. Data were expressed as the mean \pm standard error (SEM), and data analysis was performed with a statistical program (Graph Pad Prism (version 6.0), GraphPad Software, Inc., San Diego, CA, USA).

\section{Results}

\subsection{Assessment of food intake and body weight of the mother rats}

The assessment of food intake and body weight of the mother rats showed no significant difference between groups $\mathrm{CN}$ and PD during the periods of pregnancy and lactation (Fig. 1).

\subsection{Radiographic and histological examination of maternal periodontal} tissues

On radiographic and histological examination, the $\mathrm{CN}$ group exhibited characteristics consistent with a normal condition of the dental and periodontal tissues (Fig. 2A, C, and E). Radiographic examination of the PD group showed alveolar bone loss and decreased alveolar bone radiopacity in the first molar region (Fig. 2B). Histologic examination showed severe impairment in the integrity and structure of the junctional and sulcular epithelium. Significant inflammatory infiltrate, predominantly comprising composed of mononuclear cells and polymorphonuclear cells, was present in the connective tissue of the interproximal and furcation region (Fig. 2D and F). All specimens showed marked bone loss (Fig. 2B and D). The bone of the interdental and interradicular septa had a very irregular outline, numerous resorption lacunae, and fully active osteoclasts (Fig. 2F and G). Areas of external root resorption were observed in most of the specimens (Fig. 2F).

\subsection{Assessment of body weight, food intake and tissue weight in offspring}

The PD-o group had a significantly lower birth weight $(p<0.0001)$ compared to the $\mathrm{CN}-\mathrm{o}$ group (Fig. $3 \mathrm{~A}$ ). There was no difference in the mean body weight assessed weekly from after birth until 75 days of age, between the $\mathrm{CN}-\mathrm{O}$ and PD-o groups (Fig. $3 \mathrm{C}$ ). There was a significant increase $(p<0.05)$ in the absolute and relative weights of the periepididymal white adipose tissue in the PD-o group compared to the tissue weights in the CN-o group (Table 1). However, the absolute and relative weights of the GSM were significantly decreased $(p<0.05)$ in the PD-o group compared to the GSM weights in the CNo group.

The weekly evaluation of the food intake in the offspring showed that there was no difference between the $\mathrm{CN}-\mathrm{O}$ and $\mathrm{PD}-\mathrm{o}$ groups after weaning until the end of the experiment (Fig. 3B).

\subsection{Glycemia, insulinemia, HOMA-IR index, and plasma concentration of TNF- $\alpha$ of offspring}

There was no statistical difference in fasting glycemia between the groups. However, the insulinemia values were increased in the PD-o group compared to the concentrations in the $\mathrm{CN}$-o group. Furthermore, the HOMA-IR index, calculated from these parameters, was significantly higher $(p<0.05)$ in the PD-o group than the index in the CN-o group (Table 2). TNF- $\alpha$ levels were higher $(p<0.01)$ in the PD-o group compared to $\mathrm{CN}-\mathrm{o}$ group.

\subsection{Akt serine phosphorylation status of offspring}

The magnitude of Akt serine phosphorylation after insulin stimulation increased significantly from baseline in both groups in the GSM $(p<0.05)$. The Akt serine phosphorylation after $(p<0.05)$ insulin stimulation was significantly lower in the PD-o group than the phosphorylation level in the $\mathrm{CN}-\mathrm{o}$ group (Fig. 4).
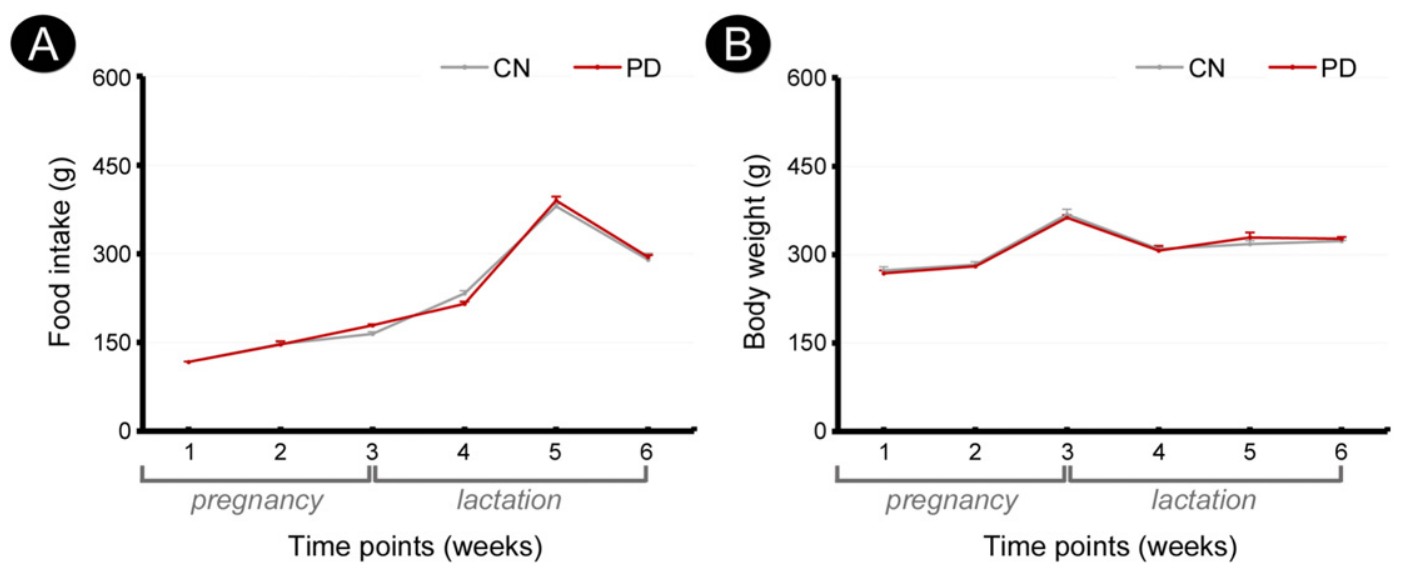

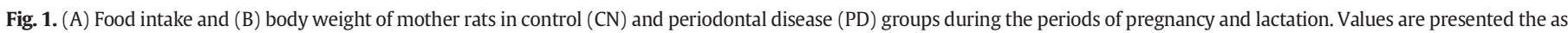
mean \pm standard error ( $n=8$ per group). 

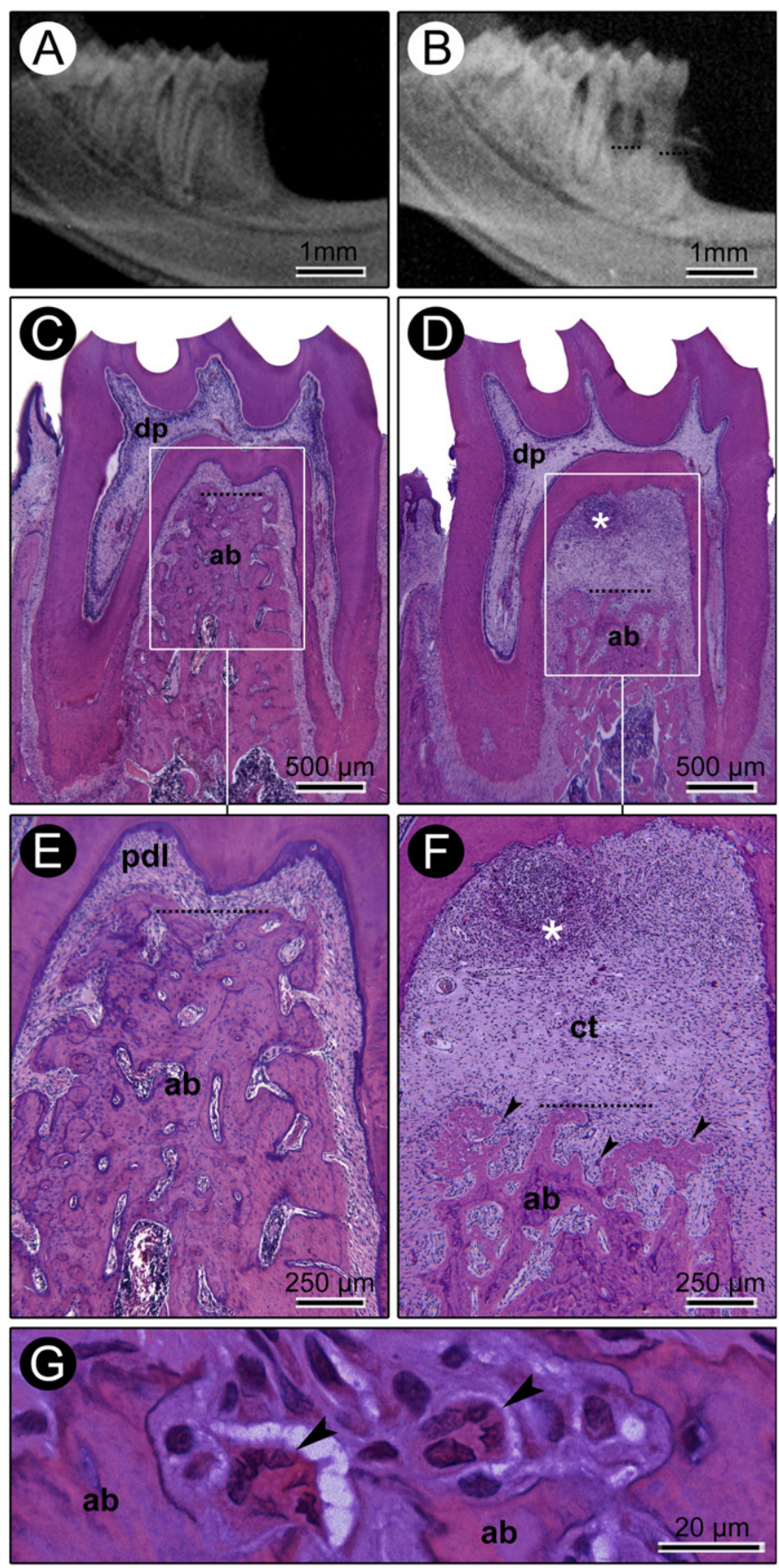

Fig. 2. Radiographs and photomicrographs of the mandibular first molars in the control (CN) (A, C and E) and periodontal disease (PD) (B, D, F and G) groups. The radiographic $(\mathrm{A})$ and histological ( $\mathrm{C}$ and $\mathrm{E}$ ) characteristics are consistent with a normal pattern in the $\mathrm{CN}$ group. In group PD it is observed persistent local inflammation and severe alveolar bone loss (B, D and F) with the presence of active osteoclasts ( $F$ and $G$ ). Abbreviations and symbols: ab, alveolar bone; arrows, osteoclasts; asterisks, inflammatory infiltrate; ct, connective tissue; dp, dental pulp; pdl, periodontal ligament. H\&E staining. Scale bars: A and D, $1 \mathrm{~mm}$; B and E, $500 \mu \mathrm{m} ; \mathrm{C}$ and F, $250 \mu \mathrm{m}$; and G, $20 \mu \mathrm{m}$.

\subsection{GLUT4 content in skeletal muscle of offspring}

Fig. 5A, B, and C shows the GLUT4 content analysis in the PM and $\mathrm{M}$, and the GLUT4 translocation index, respectively. There was no difference between the groups in $\mathrm{M}$; however, the PD-o group showed a significantly lower PM $(p<0.01)$ and translocation index $(p<0.05)$ than those in the $\mathrm{CN}-\mathrm{o}$ group.
(A)
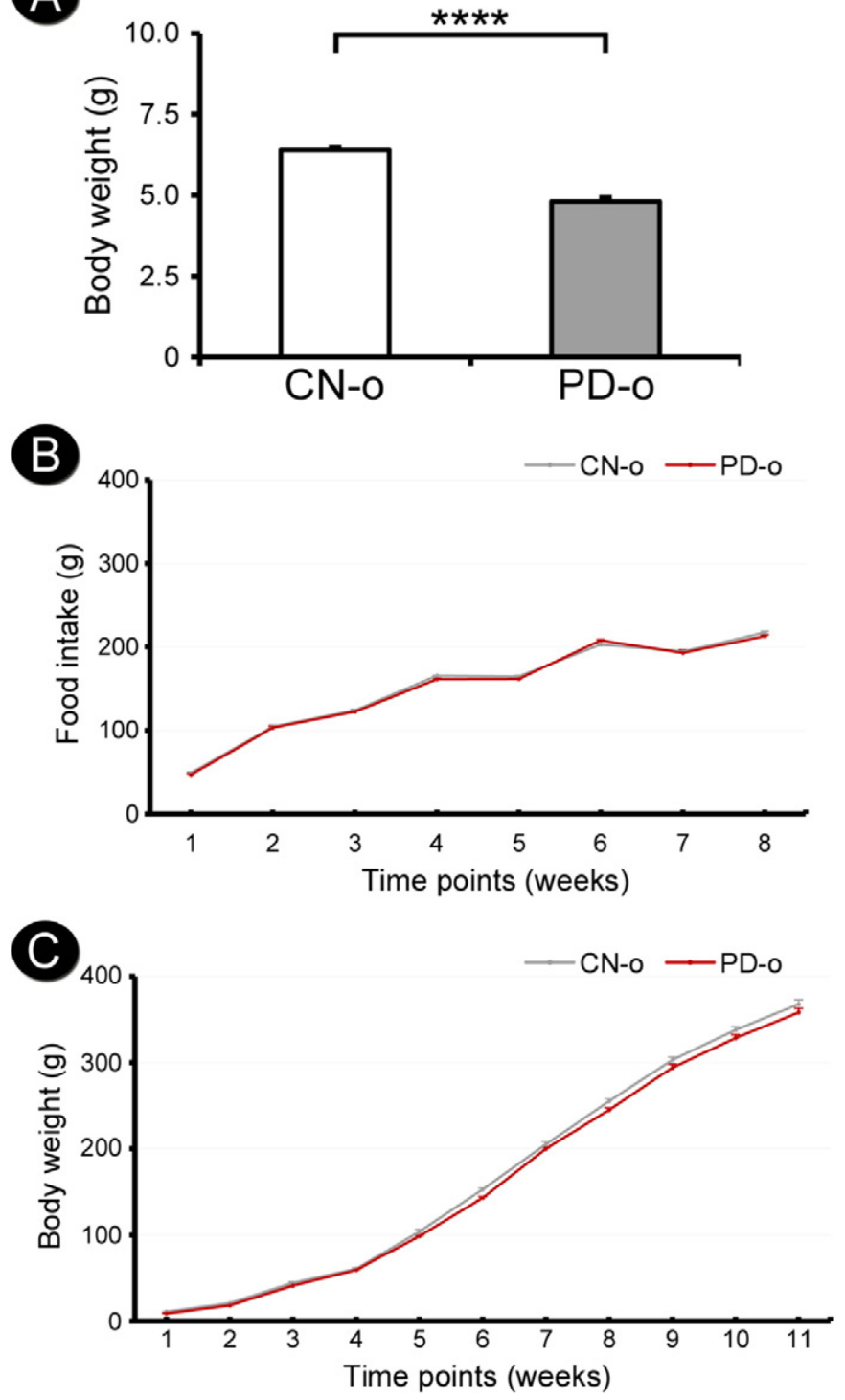

Fig. 3. (A) Body weight of offspring in the control $(\mathrm{CN}-\mathrm{O})$ and periodontal disease (PD-O) groups at birth. (B) Food intake of offspring in $\mathrm{CN}-\mathrm{O}$ and $\mathrm{PD}-\mathrm{o}$ groups assessed weekly from weaning until the end of the experiment. (C) Body weight of offspring in CN-o and PD-o groups assessed weekly from after birth until 75 days of age. Values are presented the as mean \pm standard error $\left(n=16\right.$ per group). ${ }^{* * * *} p<0.0001$

\section{Discussion}

Several studies have correlated maternal periodontal disease, LBW, and premature birth [20-22]. However, some studies show no this relationship $[12,23,24]$. Thus, our goal was to establish a relationship between maternal periodontal disease, LBW, and changes in the final stage of the insulin signaling in the muscle tissue of their offspring. The results of this study demonstrate that maternal periodontal disease causes LBW, insulin resistance, decreased in Akt serine phosphorylation, and changes in the translocation index and GLUT4 content in GSM.

LBW was observed in the experimental group. This result is consistent with those of several studies where maternal periodontal disease caused LBW [25-27]. The infection by Campylobacter rectus (the bacterium present in periodontitis) in mice induces a reduction in the size of placental labyrinth [28]. Modifications to the placental labyrinth structure may promote an insufficient supply of the fetus and thus impaired growth and LBW [29]. Additionally, in the present study, it 
Table 1

Absolute and relative weights of the periepididymal white adipose tissue and gastrocnemius skeletal muscle.

\begin{tabular}{lll}
\hline Tissue & CN-o & PD-o \\
\hline WAT g & $3.03 \pm 0.16$ & $3.82 \pm 0.22^{*}$ \\
WAT g/100 g of body weight & $0.85 \pm 0.05$ & $1.05 \pm 0.07^{*}$ \\
GSM g & $2.36 \pm 0.06$ & $2.14 \pm 0.06^{*}$ \\
GSM g/100 g of body weight & $0.65 \pm 0.02$ & $0.59 \pm 0.01^{*}$ \\
\hline
\end{tabular}

$* p<0.05$ compared with the control group. Values are presented as the mean \pm standard error $(n=8)$. CN-o, control group offspring; PD-o, periodontal disease offspring; WAT, periepididymal white adipose tissue; and GSM, gastrocnemius skeletal muscle.

should be noted that the LBW observed in the PD-o group was not due to differences in the body weight or in the food intake of the mother rats.

As noted the above, the PD-o group showed LBW, however, from the first week life the groups showed no significant difference in body weight. The results of this present study are in agreement with the phenomenon known as catch-up grown. This phenomenon suggests that infants submitted to a transient period of growth inhibition have accelerated growth, reaching similar weight and/or height to that of normal infants [30]. Moreover, the catch-up grown is associated with increased susceptibility to obesity and type 2 diabetes [31-33].

In this study, despite not find a significant difference in weight of the rat at 75 days of age when evaluating the weight of the white adipose tissue and skeletal muscle at the time of euthanasia we observed the increase in WAT weight and GSM decreased weight in the PD-the group. Although this change in GSM weight did not affect the overall body weight, according to Shirakashi et al. [12], increased in adipose tissue may have offset the decreased muscle tissue weight. Increased adipose tissue promotes increases in free fatty acids and inflammatory cytokines [34] and decreased muscle tissue is a risk for insulin resistance [35]. Moreover, the assessment of food intake in offspring showed no significant difference between the groups from weaning until the end of the experiment. Therefore, from these data we can conclude that there was no influence of food intake in the results of this study.

In the present study, we observed an increased fasting insulinemia and decreased insulin sensitivity in the PD-o group using the HOMA-IR index, and found no difference in the fasting glycemia between the groups. These results are consistent with the fetal salvage hypothesis. Children who experience intrauterine growth restriction (IUGR) develop peripheral insulin resistance through a redistribution of nutrients such as glucose favoring the essential organs, which permanently reduces the number or function of glucose transporters in skeletal muscle. As a result of this decreased peripheral insulin sensitivity, pancreatic $\beta$-cells are stimulated to produce larger amounts of insulin to achieve normal glycemia, which leads to their eventual depletion [36,37]. Additionally, studies by Amarilyo et al. [38] showed that the umbilical cord blood of children with IUGR contained high concentrations of interleukin 6 (IL-6), TNF- $\alpha$, Creactive protein, and thrombopoietin.

Table 2

Glycemia, insulinemia, HOMA-IR index, and plasma concentration TNF- $\alpha$ in offspring of control rats and offspring of rats with experimental periodontal disease.

\begin{tabular}{llc}
\hline Parameters & CN-o & PD-o \\
\hline Glycemia & $5.98 \pm 0.14$ & $6.02 \pm 0.28$ \\
Insulinemia & $9.95 \pm 1.66$ & $24.85 \pm 4.11^{* *}$ \\
HOMA-IR & $2.67 \pm 0.43$ & $6.64 \pm 1.39^{*}$ \\
TNF- $\alpha$ & $8.55 \pm 0.53$ & $10.86 \pm 0.55^{* *}$ \\
\hline
\end{tabular}

Values are presented as the mean \pm standard error ( $n=8$ per group). Plasma glucose measured in $\mathrm{mmol} / \mathrm{L}$, insulin in $\mu \mathrm{IU} / \mathrm{mL}$ and TNF- $\alpha$ in $\mathrm{pg} / \mathrm{mL}$. CN-o, control group offspring; PD-o, periodontal disease offspring; HOMA-IR, homeostasis model assessment of insulin resistance.

* $p<0.05$

** $p<0.01$
Defects in glucose regulation define type-2 diabetes [39] and inflammatory cytokines, such as TNF- $\alpha$, are elevated in the adult offspring of rats with PD [12]. These results are in agreement with this study. As described above, excessive TNF- $\alpha$ levels cause insulin resistance by modifying insulin signal transduction [9]. According to Hotamisligil et al. [40] the TNF- $\alpha$ induces serine phosphorylation of IRS- 1 and convert IRS- 1 into an inhibitor of the IR tyrosine kinase activity in vitro. The present study found LBW and decreased Akt phosphorylation status after insulin stimulation in the GSM of the PD-o group. These results are consistent with those of Jensen et al. [41] who observed that adult humans with LBW had decreased phosphorylation of proteins such as Akt at Ser 473 after insulin stimulation of muscle tissue.

According to Plomgaard et al. [9], TNF- $\alpha$ infusion in humans increases phosphorylation of p70 S6 kinase (S6K), extracellular signalregulated protein (ERK: ERK1 and ERK2), and c-Jun amino-terminal kinase (JNK), and simultaneously increases the serine phosphorylation of IRS-1. This impairs glucose uptake by decreasing the Akt substrate 160 (AS160), which is responsible for the cascade regulating GLUT4 translocation.

Insulin-mediated glucose uptake in the heart, skeletal muscle, and adipose tissue is performed by the insulin-sensitive glucose transporter, GLUT4 [42]. This uptake requires translocation of GLUT4 from the M fraction to the PM fraction, and the Akt protein is essential in mediating this process [43]. Transgenic mice lacking or overexpressing GLUT4 exhibit a decrease or increase insulin

A skeletal muscle

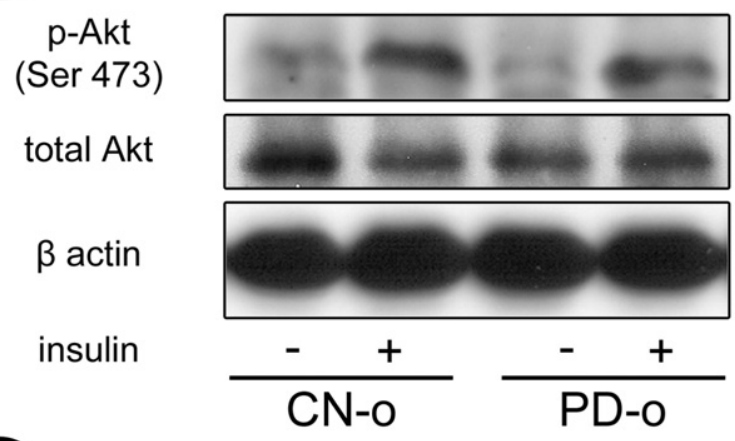

B

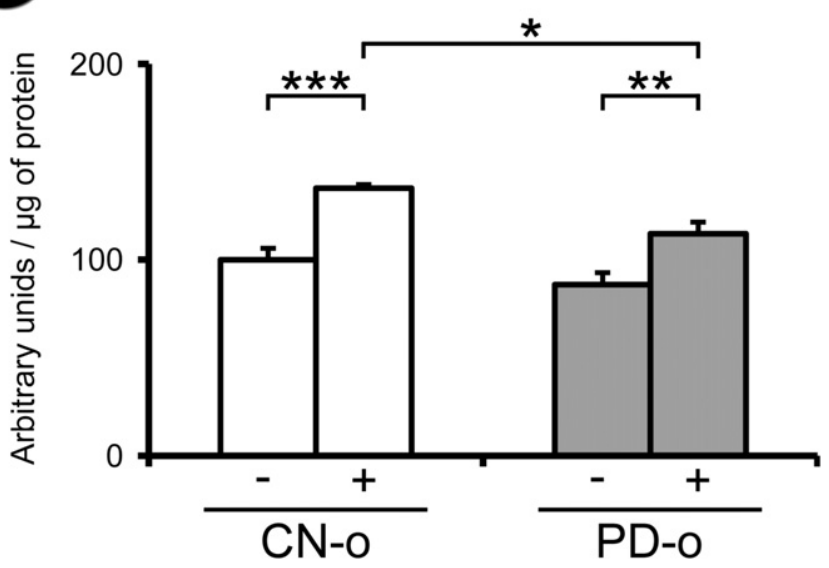

Fig. 4. Evaluation of Akt serine phosphorylation status before $(-)$ and after $(+)$ insulin stimulation in the gastrocnemius muscle of rats in the control $(\mathrm{CN}-\mathrm{O})$ and periodontal disease (PD-o) groups. (A) Typical autoradiography: equal amounts of protein were analyzed by SDS-PAGE ( $185 \mathrm{mg}$ ). $\beta$-actin and total Akt (to normalize for equal protein loading). (B) Akt serine phosphorylation status (expressed in arbitrary units) are presented as the mean \pm standard error ( $n=8$ per group). ${ }^{*} p<0.05,{ }^{* *} p<0.01$, and ${ }^{* * * *} p<0.001$ 

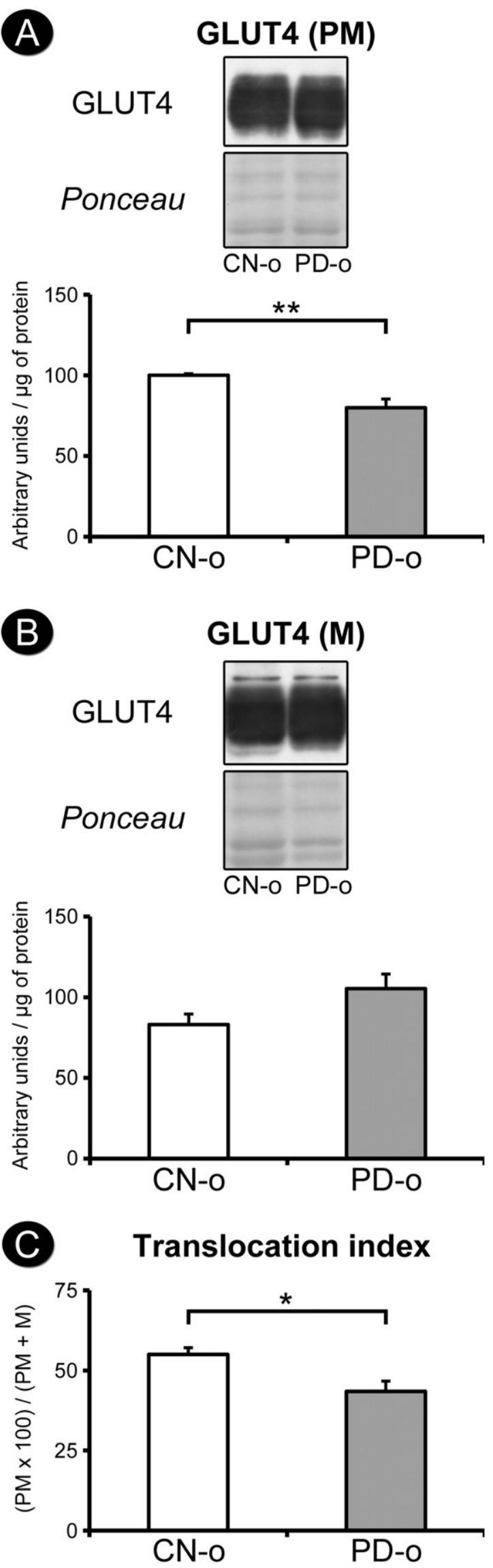

sensitivity, respectively [39,44,45]; thus, this transporter is critical in maintaining glucose homeostasis [46]. In the evaluation of the GLUT4 content of the in GSM, we observed a decrease in the PM fraction and translocation index of the PD-o group, but there was no significant difference in the $\mathrm{M}$ fraction. This can be explained by the decreased Akt serine phosphorylation within the same tissue, as this protein activation is required for GLUT4 translocation.

Using flow cytometry, Xie et al. [47] found that significantly elevated concentrations of inflammatory cytokines such as TNF- $\alpha$, interleukin-1 beta (IL-1 $\beta$ ), and IL-6 in 3T3-L1 cells (pre-adipocytes) reduced the GLUT4 expression. These cytokines play an important role in the regulation of glucose metabolism, and excessive activation of these inflammatory pathways may represent a critical step in the development of insulin resistance [48]. Ozanne et al. [49] verified that the muscle tissue of adult humans with LBW showed decreased expression of proteins within the insulin signaling pathway, including protein kinase $\mathrm{C}$ (PKC) $\zeta$, p $85 \alpha$ (regulatory subunit of PI 3-kinase), p110 $\beta$ (catalytic subunit of PI 3-kinase), and GLUT4. In another model of fetal programming, a low sodium maternal diet resulted in LBW in the offspring and increased adiposity in the adult female offspring. This finding reinforces the results of the present study in demonstrating the influence of intra-uterine disturbances on the adult offspring [50]. The results of this study emphasize the importance of addressing maternal periodontal disease in order to prevent adverse outcomes in adult offspring.

\section{Conclusions}

Maternal periodontal disease causes low birth weight and alterations in the final stage of insulin signaling in skeletal muscle of adult offspring. These findings demonstrate the impact that periodontal disease has on the intrauterine environment in the short-term, and on the long-term predisposition to certain diseases in adulthood of the offspring. This reinforces the importance of maintaining maternal oral health in ensuring the overall health of the offspring.

\section{Acknowledgments}

This study was supported by grants \#2013/08783-4 from the São Paulo Research Foundation (FAPESP), São Paulo, SP, Brazil, ProRectory of Research of UNESP (PROPe-UNESP): 2077/002/14$\mathrm{PROPe} / \mathrm{CDC}$ and Foundation for the Development of UNESP (FUNDUNESP): 328/2007-DFP. We thank Associate Professor Dr. Guilherme de Paula Nogueira and laboratory technician, Mrs. Devani Mariano Pinheiro (Department of Support, Animal Production and Health, Veterinary Medicine School of Araçatuba, São Paulo State University - UNESP, Brazil), for help in the insulinemia evaluation.

\section{References}

[1] D. Lin, M.A. Smith, C. Champagne, J. Elter, J. Beck, S. Offenbacher, Porphyromonas gingivalis infection during pregnancy increases maternal tumor necrosis factor alpha, suppresses maternal interleukin-10, and enhances fetal growth restriction and resorption in mice, Infect. Immun. 71 (2003) 5156-5162.

[2] E. Ercan, K. Eratalay, O. Deren, D. Gur, O. Ozyuncu, B. Altun, C. Kanli, P. Ozdemir, H. Akincibay, Evaluation of periodontal pathogens in amniotic fluid and the role of periodontal disease in pre-term birth and low birth weight, Acta Odontol. Scand. 71 (2013) 553-559.

[3] C.N. Hales, D.J. Barker, P.M. Clark, L.J. Cox, C. Fall, C. Osmond, P.D. Winter, Fetal and infant growth and impaired glucose tolerance at age 64, BMJ 303 (1991) 1019-1022.

[4] D.J. Barker, The long-term outcome of retarded fetal growth, Clin. Obstet. Gynecol. 40 (1997) 853-863.

Fig. 5. GLUT4 content in the plasma membrane (PM) (A) and microsomal-enriched (M) (B) fractions of the gastrocnemius muscle. The top shows representative images of GLUT4 content in the PM (A) and M (B) and the respective loading controls. (C) GLUT4 translocation index in the offspring of the control $(\mathrm{CN}-\mathrm{O})$ and experimental periodontal disease (PD-o) groups. The results are presented as mean \pm standard error $(n=6$ per group). ${ }^{*} p<0.05,{ }^{* *} p<0.01$ 
[5] D.J. Barker, J.G. Eriksson, T. Forsén, C. Osmond, Fetal origins of adult disease: strength of effects and biological basis, Int. J. Epidemiol. 31 (2002) 1235-1239.

[6] D.J. Barker, Fetal origins of coronary heart disease, BMJ 311 (1995) 171-174.

[7] S.C. Langley-Evans, S. McMullen, Developmental origins of adult disease, Med. Princ. Pract. 19 (2010) 87-98.

[8] T. Sert, F.Y. Kırzıoğlu, O. Fentoğlu, F. Aylak, T. Mungan, Serum placental growth factor, vascular endothelial growth factor, soluble vascular endothelial growth factor receptor-1 and -2 levels in periodontal disease, and adverse pregnancy outcomes, J. Periodontol. 82 (2011) 1735-1748.

[9] P. Plomgaard, K. Bouzakri, R. Krogh-Madsen, B. Mittendorfer, J.R. Zierath, B.K. Pedersen, Tumor necrosis factor-alpha induces skeletal muscle insulin resistance in healthy human subjects via inhibition of Akt substrate 160 phosphorylation, Diabetes 54 (2005) 2939-2945.

[10] P. Bevan, Insulin signalling, J. Cell Sci. 114 (2001) 1429-1430.

[11] A.F. Rowland, D.J. Fazakerley, D.E. James, Mapping insulin/GLUT4 circuitry, Traffic 12 (2011) 672-681.

[12] D.J. Shirakashi, R.P. Leal, N.H. Colombo, F.Y. Chiba, C.A. Garbin, E.G. Jardim, C. Antoniali, D.H. Sumida, Maternal periodontal disease in rats decreases insulin sensitivity and insulin signaling in adult offspring. J. Periodontol. 84 (2013) 407-414.

[13] C.O. Rodini, A.C. Batista, T.J. Dionísio, C.F. Santos, F.Q. Cunha, V.S. Lara, Morphologic evaluation and expression of matrix metalloproteinases-2 and 9 and nitric oxide during experimental periodontal disease in rat, J. Mol. Histol. 39 (2008) 275-282.

[14] V.G. Garcia, V.C. Novaes, J.M. de Almeida, M. Longo, E. Ervolino, S.R. Bomfim, L.H. Theodoro, Evaluation of the progression and treatment of experimental periodontitis in rats subjected to chemotherapy with 5-fluorouracil, Support. Care Cancer 23 (2014) 2007-2017.

[15] E. Bonora, G. Targher, M. Alberiche, R.C. Bonadonna, F. Saggiani, M.B. Zenere, T. Monauni, M. Muggeo, Homeostasis model assessment closely mirrors the glucose clamp technique in the assessment of insulin sensitivity: studies in subjects with various degrees of glucose tolerance and insulin sensitivity, Diabetes Care 23 (2000) 57-63.

[16] N.N. Lasheen, Pancreatic functions in high salt fed female rats, Physiol. Rep. 3 (2015), e12443.

[17] C.R. Carvalho, S.L. Brenelli, A.C. Silva, A.L. Nunes, L.A. Velloso, M.J. Saad, Effect of aging on insulin receptor, insulin receptor substrate-1, and phosphatidylinositol 3kinase in liver and muscle of rats, Endocrinology 137 (1996) 151-159.

[18] Y. Mitsumoto, A. Klip, Development regulation of the subcellular distribution and glycosylation of GLUT1 and GLUT4 glucose transporters during myogenesis of L6 muscle cells, J. Biol. Chem. 267 (1992) 4957-4962.

[19] M. Dolnikoff, A. Martín-Hidalgo, U.F. Machado, F.B. Lima, E. Herrera, Decreased lipolysis and enhanced glycerol and glucose utilization by adipose tissue prior to development of obesity in monosodium glutamate (MSG) treated-rats, Int. J. Obes. Relat. Metab. Disord. 25 (2001) 426-433.

[20] S. Offenbacher, V. Katz, G. Fertik, J. Collins, D. Boyd, G. Maynor, R. McKaig, J. Beck, Periodontal infection as a possible risk factor for preterm low birth weight, J. Periodontol. 67 (1996) 1103-1113.

[21] Y.S. Khader, Q. Ta'ani, Periodontal diseases and the risk of preterm birth and low birth weight: a meta-analysis, J. Periodontol. 76 (2005) 161-165.

[22] A.N. Guimarães, A. Silva-Mato, L.O. Miranda Cota, F.M. Siqueira, F.O. Costa, Maternal periodontal disease and preterm or extreme preterm birth: an ordinal logistic regression analysis, J. Periodontol. 81 (2010) 350-358.

[23] E.S. Davenport, C.E. Williams, J.A. Sterne, S. Murad, V. Sivapathasundram, M.A. Curtis, Maternal periodontal disease and preterm low birthweight: case-control study, J. Dent. Res. 81 (2002) 313-318.

[24] J.I. Ryu, K. Oh, H. Yang, B.K. Choi, J.E. Ha, B.H. Jin, H.D. Kim, K.H. Bae, Health behaviors, periodontal conditions, and periodontal pathogens in spontaneous preterm birth: a case-control study in Korea, J. Periodontol. 81 (2010) 855-863.

[25] S. Mannem, V.K. Chava, The relationship between maternal periodontitis and preterm low birth weight: a case-control study, Contemp. Clin. Dent. 2 (2011) 88-93.

[26] A.N. Guimarães, A. Silva-Mato, F.M. Siqueira, R.M. Cyrino, L.O. Cota, F.O. Costa, Very low and low birth weight associated with maternal periodontitis, J. Clin. Periodontol. 39 (2012) 1024-1031.

[27] A. Haerian-Ardakani, Z. Eslami, F. Rashidi-Meibodi, A. Haerian, P. Dallalnejad, M. Shekari, A. Moein Taghavi, S. Akbari, Relationship between maternal periodontal disease and low birth weight babies, Iran, J. Reprod. Med. 11 (2013) 625-630.
[28] S. Offenbacher, E.L. Riché, S.P. Barros, Y.A. Bobetsis, D. Lin, J.D. Beck, Effects of maternal Campylobacter rectus infection on murine placenta, fetal and neonatal survival, and brain development, J. Periodontol. 76 (2005) 2133-2143.

[29] P.N. Madianos, Y.A. Bobetsis, S. Offenbacher, Adverse pregnancy outcomes (APOs) and periodontal disease: pathogenic mechanisms, J. Clin. Periodontol. 40 (2013) S170-S180.

[30] B. Boersma, J.M. Wit, Catch-up growth, Endocr. Rev. 18 (1997) 646-661.

[31] N. Cameron, E.W. Demerath, Critical periods in human growth and their relationship to diseases of aging, Am. J. Phys. Anthropol. 35 (2002) 159-184.

[32] K.K. Ong, C.J. Petry, P.M. Emmett, M.S. Sandhu, W. Kiess, C.N. Hales, A.R. Ness, D.B. Dunger, ALSPAC study team, insulin sensitivity and secretion in normal children related to size at birth, postnatal growth, and plasma insulin-like growth factor-I levels, Diabetologia 47 (2004) 1064-1070.

[33] J.C. Jimenez-Chillaron, M. Hernandez-Valencia, A. Lightner, R.R. Faucette, C. Reamer R. Przybyla, S. Ruest, K. Barry, J.P. Otis, M.E. Patti, Reductions in caloric intake and early postnatal growth prevent glucose intolerance and obesity associated with low birthweight, Diabetologia 49 (2006) 1974-1984.

[34] G. Boden, Obesity and free fatty acids, Endocrinol. Metab. Clin. N. Am. 37 (2008) 635-646 viii-ix.

[35] C.N. Hales, D.J. Barker, The thrifty phenotype hypothesis, Br. Med. Bull. 60 (2001) 5-20.

[36] P.L. Hofman, W.S. Cutfield, E.M. Robinson, R.N. Bergman, R.K. Menon, M.A. Sperling P.D. Gluckman, Insulin resistance in short children with intrauterine growth retardation, J. Clin. Endocrinol. Metab. 82 (1997) 402-406.

[37] S. Cianfarani, D. Germani, F. Branca, Low birthweight and adult insulin resistance: the "catch-up growth" hypothesis, Arch. Dis. Child. Fetal Neonatal Ed. 81 (1999) F71-F73.

[38] G. Amarilyo, A. Oren, F.B. Mimouni, Y. Ochshorn, V. Deutsch, D. Mandel, Increased cord serum inflammatory markers in small-for-gestational-age neonates, J. Perinatol. 31 (2011) 30-32.

[39] E.D. Berglund, C.Y. Li, J.E. Ayala, O.P. McGuinness, D.H. Wasserman, Regulation of endogenous glucose production in glucose transporter 4 over-expressing mice, PLoS One 7 (2012), e52355.

[40] G.S. Hotamisligil, P. Peraldi, A. Budavari, R. Ellis, M.F. White, B.M. Spiegelman, IRS-1mediated inhibition of insulin receptor tyrosine kinase activity in TNF-alpha- and obesity-induced insulin resistance, Science 271 (1996) 665-668.

[41] C.B. Jensen, M.S. Martin-Gronert, H. Storgaard, S. Madsbad, A. Vaag, S.E. Ozanne Altered PI3-kinase/Akt signalling in skeletal muscle of young men with low birth weight, PLoS One 3 (2008), e3738.

[42] S. Huang, M.P. Czech, The GLUT4 glucose transporter, Cell Metab. 5 (2007) 237-252.

[43] G.I. Welsh, I. Hers, D.C. Berwick, G. Dell, M. Wherlock, R. Birkin, S. Leney, J.M. Tavaré, Role of protein kinase B in insulin-regulated glucose uptake, Biochem. Soc. Trans. 33 (2005) 346-349.

[44] E.B. Katz, R. Burcelin, T.S. Tsao, A.E. Stenbit, M.J. Charron, The metabolic consequences of altered glucose transporter expression in transgenic mice, J. Mol. Med. (Berl.) 74 (1996) 639-652.

[45] E. Carvalho, K. Kotani, O.D. Peroni, B.B. Kahn, Adipose-specific overexpression of GLUT4 reverses insulin resistance and diabetes in mice lacking GLUT4 selectively in muscle, Am. J. Physiol. Endocrinol. Metab. 289 (2005) E551-E561.

[46] N.M. Leguisamo, A.M. Lehnen, U.F. Machado, M.M. Okamoto, M.M. Markoski, G.H. Pinto, B.D. Schaan, GLUT4 content decreases along with insulin resistance and high levels of inflammatory markers in rats with metabolic syndrome, Cardiovasc. Diabetol. 11 (2012) 100.

[47] L. Xie, M.T. Ortega, S. Mora, S.K. Chapes, Interactive changes between macrophages and adipocytes, Clin. Vaccine Immunol. 17 (2010) 651-659.

[48] K.E. Wellen, G.S. Hotamisligil, Inflammation, stress, and diabetes, J. Clin. Invest. 115 (2005) 1111-1119.

[49] S.E. Ozanne, C.B. Jensen, K.J. Tingey, H. Storgaard, S. Madsbad, A.A. Vaag, Low birthweight is associated with specific changes in muscle insulin-signalling protein expression, Diabetologia 48 (2005) 547-552.

[50] K.L. Lopes, L.N. Furukawa, I.B. de Oliveira, M.S. Dolnikoff, J.C. Heimann, Perinatal salt restriction: a new pathway to programming adiposity indices in adult female Wistar rats, Life Sci. 82 (2008) 728-732. 